\title{
EFEITOS DO EXERCÍCIO FÍSICO EM INDIVÍDUOS HIPERTENSOS DA UNIDADE BÁSICA DE SAÚDE DO OESTE PAULISTA
}

Elaine Ayumi Honda, Ana Clara Campagnolo Gonçalves Toledo, Francis Lopes Pacagnelli, Ana Karênina Dias De Almeida Sabela, Thainá Aparecida Amaral Magosso, Priscila Figueiredo Correia, Renata Augusta Beloni Digiovani

Universidade do Oeste Paulista - UNOESTE, Faculdade de Fisioterapia, Presidente Prudente/SP. E-mail: elaine.a.honda@gmail.com.

\section{RESUMO}

A hipertensão arterial (HA) é uma patologia multifatorial caracterizada por níveis pressóricos elevados e sustentados de $\geq 140 / 90 \mathrm{mmHg}$, associada a distúrbios metabólicos, alterações funcionais e ou estruturais, sendo agravadas na presença de fatores de risco como obesidade, tabagismo e diabetes melito. $O$ objetivo do estudo foi verificar a influência do exercício físico no impacto da saúde de frequentadores de um Programa de HAS da Unidade Básica de Saúde da região do Oeste Paulista. Realizado a aferição da pressão arterial e foi observado que a pressão estava controlada. Concluímos que o exercício físico colabora a manutenção da pressão arterial e na prevenção e conscientização dos danos à saúde.

Palavras-chave: hipertensão, exercício físico, obesidade, fatores de risco, doenças cardiovasculares.

\section{EFFECTS OF PHYSICAL EXERCISE ON HYPERTENSIVE INDIVIDUALS OF THE BASIC HEALTH UNITOF OESTE PAULISTA PHYSICAL EXERCISE ON HYPERTENSIVE}

\begin{abstract}
Hypertension ( $\mathrm{AH}$ ) is a multifactorial pathology characterized by elevated and sustained blood pressure levels of $\geq 140 / 90 \mathrm{mmHg}$, associated with metabolic disturbances, functional and structural alterations, and is aggravated by the presence of risk factors such as obesity, smoking and diabetes mellitus. The objective is to verify the influence of physical exercise on the health impact of attendees of a Health Program of the Basic Health Unit of the region of Oeste Paulista. Blood pressure was measured and pressure was observed. We conclude that physical exercise contributes to the maintenance of blood pressure and to prevention and a wareness of health damage.
\end{abstract}

Keywords: hypertension, physicalexercise, obesity, riskfactors, cardiovascular diseases 


\section{INTRODUÇÃO}

A Hipertensão Arterial Sistêmica (HAS) é uma patologia multifatorial caracterizada por níveis pressóricos elevados e sustentados de $\geq 140 / 90 \mathrm{mmHg}$. Está frequentemente associada a distúrbios metabólicos, alterações funcionais e ou estruturais, sendo agravadas na presença de fatores de risco como obesidade, tabagismo, dislipidemia e diabetes melito. É uma condição clínica de início assintomático, não possui atuação isolada, o que dificulta o diagnóstico precoce, além de manter associações com complicações secundárias como acidente vascular encefálico, morte súbita e infarto agudo do miocárdio ${ }^{1}$.

Segundo os dados epidemiológicos sobre a temática, cerca de 7,6 milhões de pessoas morrem ao ano em todo o mundo e atinge com maior frequência a faixa etária entre 45 e 69 anos. Estima-se que $80 \%$ desses óbitos ocorrem em países emergentes como Brasil. O sedentarismo é um fator que está relacionado a obesidade e predispõe indivíduos à hipertensão, estudos mostram que a prática regular de exercícios físicos causam impactos positivos nos níveis pressóricos com redução ou normalização dos mesmos, além da redução de peso e dos fatores de risco cardiovasculares ${ }^{2}$.

Segundo Lessa ${ }^{3}$ (2001) ressaltou prevalência de HAS elevada entre o adultos de raça negra. Evidenciou predomínio de até $130 \%$ entre as mulheres negras comparada às caucasianas. Em estudos, salientam-se que os fatores que corroboram a prevalência de HAS na população afrodescendentes estão associados a predisposição genética, ao acesso restrito aos serviços de saúde e ao estresse da discriminação racial.

Desta forma o exercício físico e bons hábitos alimentares são recursos eficazes na redução dos riscos cardiovasculares e proporciona a melhora no condicionamento físico, diminuição de massa gorda, força muscular, coordenação, equilíbrio e refletindo na redução da pressão arterial sistêmica (PAS). ${ }^{4,5}$ Ocasionando a mudança no estilo de vida, controle de estresse e demais fatores que irão promover a redução de complicações futuras e controle dos níveis pressóricos dentro da normalidade. ${ }^{5,6}$

Este estudo tem como objetivo relacionar a prevalência dos níveis pressóricos de hipertensos com a prática regular do exercício físico, com o intuito de caracterização da amostra, verificando a influência do exercício físico no impacto da saúde deste indivíduos frequentadores de um Programa de HAS da Unidade Básica de Saúde da região do Oeste Paulista.

\section{MÉTODOS}

Refere-se de um estudo transversal, de base populacional, onde foram escolhidos aleatoriamente 16 participantes para a pesquisa, que são frequentadores do Programa de HAS da UBS da região do Oeste Paulista.

Os critérios de inclusão foram: pacientes na faixa etária entre 30 à 60 anos, não tabagista, ingestão correta dos medicamentos habituais e frequentar a unidade básica de saúde e que concorde em participar da pesquisa por meio da assinatura do Termo de Consentimento Livre Esclarecido. E os critérios de exclusão foram: possuir hipotensão, não seguir corretamente a ingestão dos medicamentos habituais, tontura e mal- estar.

A avaliação foi realizada no período da manhã e os instrumentos utilizados para a coleta de dados foram: esfigmomanometro (Premium G - tech), estetoscópio(Premium).

A pressão arterial foi aferida com o participante sentado em uma cadeira confortavelmente por pelo menos 5 minutos em repouso, com os pés apoiados no chão, em ambiente tranquilo e com o braço a ser aferido a pressão arterial, posicionado ao nível do coração. ${ }^{7,8,9}$

Para a análise dos resultados foi confeccionado um banco de dados eletrônicos. Os resultados foram expressos em média, desvio padrão e percentuais. Foram confeccionados gráficos e tabelas para a demonstração dos resultados. 
O projeto foi aprovado pelo Comitê de Ética, № CAAE 57438216.9.0000.5515 em pesquisa e está de acordo com a Resolução 466/2012 CONEP. Todos os participantes assinaram o Termo de Consentimento Livre e Esclarecido.

\section{RESULTADOS}

Foram avaliados 16 pacientes da UBS, com diagnóstico de HAS. Todos mulheres com idade média de $58 \pm 4,24$ anos, a raça da amostra foi de $68,75 \%$ são brancas, $18,75 \%$ são negras e $12,5 \%$ são pardas (Tabela 1). Em relação a profissão, 56,25\% são do lar e com relação ao estado civil $56,25 \%$ são casadas, $18,75 \%$ são divorciadas e $12,5 \%$ são solteiras e viúvas (Tabela 1 ). Observamos pressão arterial sistólica $135 \pm 7,07 \mathrm{mmHg}$ e a pressão arterial diastólica $65 \pm 7,07 \mathrm{mmHg}$, (Tabela 2). Os medicamentos utilizados foram Losartana, Hidroclorotiazida, Atenolol, Pressomede e Besilato de Anlodipino.

Tabela 1. Caracterização da amostra.

\begin{tabular}{lc}
\hline Variáveis & $\mathbf{N}=\mathbf{1 6}$ \\
\hline Idade (anos) & $58 \pm 4,24$ \\
Raça, $\mathbf{n}(\%)$ & \\
Branco & $11(68,75)$ \\
Negro & $3(18,75)$ \\
Amarelo & $0(0,00)$ \\
Pardo & $2(12,50)$ \\
Estado civil, $\mathbf{n}(\%)$ & \\
Solteira & $2(12,50)$ \\
Viúva & $2(12,50)$ \\
Casada & $9(56,25)$ \\
Divorciada & $3(18,75)$ \\
Profissão, $\mathbf{n}(\%)$ & \\
Aposentado & $7(43,75)$ \\
Do lar & $9(56,25)$ \\
\hline \hline
\end{tabular}

Valores expressos em média desvio padrão e em porcentagem (\%)

Tabela 2. Dados cardiovasculares.

\begin{tabular}{ll}
\hline Parâmetros pressóricos & Média \pm desvio padrão \\
\hline PAS (mmHg) & $135 \pm 7,04$ \\
PAD (mmHg) & $65 \pm 7,07$ \\
\hline \hline
\end{tabular}

PAS: Pressão Arterial Sistólica; PAD: Pressão Arterial Diastólica

\section{DISCUSSÃO}

No presente estudo teve predominância de mulheres, uma vez que é uma população que cuida da saúde comparado aos homens ${ }^{10}$. Os dados obtidos foram que a pressão arterial dessas mulheres estavam controladas preconizado em diretrizes. A amostra estudada praticam as atividades para hipertensos na UBS, três vezes semanais, com duração de 60 minutos, sendo que 10 minutos de alongamento e 50 minutos de exercício aeróbico, com intensidade moderada e não fazem acompanhamento nutricional. Entretanto, tem orientações sobre a doença, campanha de prevenção e como enfrentar essa situação, na unidade pela equipe multidisciplinar. 
A prática regular do exercício físico proporciona reduções na pressão arterial, da morbidade cardiovascular e melhora da qualidade de vida, pois uma vida ativa previne diversas doenças de ordem física quanto psicológica. Por consequência, reduz o uso de medicamentos, da dose ou a quantidade de anti- hipertensivos, além de favorecer na melhora da capacidade funcional e no prognóstico de doenças. ${ }^{11}$

A adesão à prática de exercício físico promove adaptações significativas que irão refletir na normalização dos níveis pressóricos dos indivíduos hipertenso, além de servir como estratégia para modificar o estilo de vida, tornando uma ferramenta essencial para a prevenção, tratamento e controle de hipertensão arterial. ${ }^{11}$

No estudo de Miranda e colaboradores ${ }^{12}(2017)$, envolvendo adultos hipertensos e/ou diabéticos participaram de um programa de exercício físico composta por caminhadas, exercício de alongamentos e de força com duração de 13 semanas, frequência de 3 treinos de $1 \mathrm{~h}$ por semana, observaram redução significativa na massa corporal e melhora da flexibilidade. Os autores concluíram que o programa não foi capaz de reduzir os níveis glicêmicos e pressóricos. Este estudo não corrobora com os nossos achados, pois evidenciamos que na amostra, os níveis pressóricos estavam controlados.

O estudo apresenta limitações, com um pequeno tamanho amostral, pois alguns participantes não compareceram no dia da coleta de dados, mesmo sendo divulgado o trabalho com antecedância na unidade a realização da pesquisa.

\section{CONCLUSÃO}

Concluímos que o exercício físico regular foi capaz de promover a normalização dos níveis pressóricos das participantes.

Reforçamos a importância de melhorar as orientações sobre os benefícios do exercício físico no controle da hipertensão arterial e na prevenção e redução das complicações secundárias com estratégias diversificadas para modificar este cenário.

\section{CONFLITO DE INTERESSE}

Os autores declaram não haver qualquer conflito de interesse que posso interferir neste trabalho científico.

\section{REFERÊNCIAS BIBLIOGRÁFICAS}

1. Sociedade Brasileira de Cardiologia - SBC. 7ํ Diretriz Brasileira de Hipertensão Arterial. 2016

2. Garcia EB, Takahashi C, Ribeiro F, Gutierrez M, Sonoda BR, Vanzella ML, et al. Análise da presença de obesidade, comportamentos e fatores de risco cardiovascular em indivíduos hipertensos. Colloq Vitae. 2016; 8(2)

3. Lessa I. Epidemiologia da hipertensão arterial sistêmica e insuficiência cardíaca no Brasil. RevBrasHipertens. 2001;8(4):384-92.

4. Chiara V, Sichieri R, Martins, PD. Sensibilidade e especificidade de classificação de sobrepeso em adolescentes. Rev de Saúde Pública. 2003; 2(37), 226-231, https://doi.org/10.1590/S003489102003000200010 .

5. Sousa FT. Inatividade física em universitários brasileiros: uma revisão sistemática. RevBras de Ciências da Saúde. 2011; 9(29). 
6. Mio JR. V Diretrizes Brasileira de Hipertensão Arterial. 2006.

7. Lopes AA, Hornbuckle K, James SA, Port FK. The Joint effectofraceand age ontheriskofendstage renal diseaseattributedtohypertension. Am J KidneyDis. 1994; 24: 554-60, https://doi.org/10.1016/S0272-6386(12)80211-3.

8. Sociedade Brasileira de Cardiologia - SBC. V Diretrizes Brasileiras de Hipertensão Arterial. 2007.

9. Martins CCM, Ricarte FI, Rocha LHC, Maia BR, Silva BV, Veras BA, et al.Pressão Arterial, excesso de peso e nivel de atividade física em estudante de Universidade Pública. ArqBrasCardiol. 2010; 95(2): 192- 199, https://doi.org/10.1590/S0066-782X2010005000069.

10. Fernandes PN, Bezerra MRC, Neto SJ, Batista MCV Pedrosa MLCC. A prática do exercício físico para melhoria da qualidade de vida e controle da hipertensão arterial na terceira idade.RevCiênc Saúde Nova Esperança. 2013:11(3):60-6.

11. Gobbi S; Oliani MM; Lopes GA; Coutinho FG; Bucken TL; Costa RLJ. Validade do teste de andar 6 minutos para idosas em relação ao tempo de fadiga. RevBrasAtivFís\& Saúde. 2004.

12. Miranda E, Ferreira A, Montserrat P, Oliveira G. Efeitos de um programa de exercício físico ao ar livre em diabéticos e hipertensos atendidos em um Núcleo de Apoio à Saúde da Familia de Lagoa da Prata MG. RevBrasPresc e Fisio do Exerc.2017; 11(65): 164-172 